\title{
Investigation of an IC Engine Intake Flow Based on Highly Resolved LES and PIV
}

\author{
Stefan Buhl ${ }^{1 *}$, Frank Hartmann ${ }^{1 *}$, Sebastian A. Kaiser ${ }^{2}$ and Christian Hasse ${ }^{1}$ \\ ${ }^{1}$ IEC - Department of Energy Process Engineering and Chemical Engineering, TU Bergakademie Freiberg, Reiche Zeche, \\ Fuchsmühlenweg 9, 09599 Freiberg - Germany \\ 2 Institut für Verbrennung und Gasdynamik - Reaktive Fluide, Universität Duisburg-Essen, Lotharstr. 1, 47057 Duisburg - Germany \\ e-mail: stefan.buhl@iec.tu-freiberg.de - frank.hartmann@iec.tu-freiberg.de \\ * Corresponding author
}

\begin{abstract}
To reduce emissions and fuel consumption, the current generation of gasoline engines uses technologies such as direct injection, downsizing and supercharging. All of them require a strong vortical in-cylinder charge motion, usually described as "tumble", to improve fuel-air mixing and enhance flame propagation. The tumble development strongly depends on the flow field during the intake stroke. This flow field is dominated by the intake jet, which has to be captured well in the simulation. This work investigates the intake jet on a steady-state flow bench, especially in the vicinity of the intake valve. At first, the general flow dynamics of the intake jet for three different valve lifts and three different mass flows were investigated experimentally. For the smallest valve lift (3 mm), flow-field measurements using Particle Image Velocimetry (PIV) show that the orientation of the intake jet significantly depends on the air flow rate, attaching to the pent roof for low flow rates. This phenomenon is less pronounced for higher valve lifts. An intermediate valve lift and flow rate were chosen for further investigations by scale-resolving simulations. Three different meshes (coarse, medium and fine) and two turbulence models (Sigma and Detached Eddy Simulation-Shear Stress Transport (DES-SST)) are applied to consider their effect on the numerical results. An ad-hoc postprocessing methodology based on the ensemble-averaged velocity field is presented capturing the jet centerline's mean velocity and velocity fluctuations as well as its orientation, curvature and penetration depth. The simulation results are compared to each other as well as to measurements by PIV.
\end{abstract}

\section{INTRODUCTION}

During the last few decades, regulations in terms of emission and fuel consumption have become very strict for Internal Combustion (IC) engines. To fulfill the requirements, modern spark-ignited IC engines are based on direct injection, downsizing and supercharging. These technologies require a significant in-cylinder charge motion (usually described as "tumble"), which is generated during the intake stroke. This tumble motion decreases during the compression stroke until the tumble breaks down. The resulting turbulent fluctuations increase the turbulent burning velocity, which improves the combustion process and the engine efficiency. A more detailed overview of the importance of the tumble and its influence on subsequent processes (e.g. fuel-air mixing and flame propagation) is described in $[1,2]$. In several studies it was shown that the momentum introduced by the intake jet is the main contributor to the large-scale tumble [3-5]. Due to this strong dependency on the intake jet, the intake port is optimized intensively during the engine design process to obtain a distinctive tumble motion throughout the intake and compression stroke. Often, this optimization process starts with numerical investigations of a simplified engine 
configuration on a steady-state flow bench. Despite the simplicity of the flow bench setup, a highly complex flow field emerges in the close vicinity of the intake valve and the upper part of the cylinder, which includes a multitude of phenomena:

(A) vortex shedding/turbulent flow behind the valve shaft;

(B) flow separation;

(C) turbulent intake jet;

(D) recirculation zone;

(E) wall reattachment.

Figure 1 illustrates these phenomena in the valve center plane, and introduces labels, which are referred to throughout this work. Most of these individual phenomena have been investigated in generic test cases. For example, the flow around bluff bodies [6-8] leads to a flow structure similar to A. The backward-facing step [9-11] and the turbulent offset jet $[12,13]$ include phenomena B, C, D and E. Turbulent jets (C) were investigated in $[14,15]$. Several studies showed that Scale-Resolving Simulations (SRS) can capture the flow field correctly [16-20], considering a reduced number of phenomena in simple geometries. In contrast, a previous study on the flow bench identified discrepancies between Computational Fluid Dynamics (CFD) and Particle Image Velocimetry (PIV) [21], attributable to the complexity of the flow field. This motivated further studies.

In this work, the velocity field on the valve center plane is analyzed with a focus on the flow close to the intake valve. The terminology "intake jet" $\left(C_{L}\right.$ and $\left.C_{R}\right)$ is used for the region with high velocities within and downstream of the valve gap. Starting with a maximum value within the valve gap, the high momentum of the intake jet is transported into the cylinder, spreads out and continuously slows down with increasing distance from the valve. A similar definition was used in [22], where magnetic resonance velocimetry was employed to investigate a steady-state flow bench configuration within the entire volume. Other studies investigated the intake jet on the engine symmetry plane [23-25]. Due to the location, the characteristic of the intake jet strongly differs from those in this work. Nevertheless, its definition is quite similar and focusses on the region with high velocities, which form the large-scale tumble motion.

The presented simulations are based on three different meshes (coarse, medium and fine) and two different turbulence models (Sigma and Detached Eddy Simulation-Shear Stress Transport (DES-SST)). First, the methodology to investigate the intake jet is shown, followed by a description of experimental and numerical setup. Next, an overview of the general flow topology is given. The numerical results are compared to each other and to PIV measurements. Finally, the ensemble-averaged and fluctuating velocities along the jet centerline as well as its orientation,

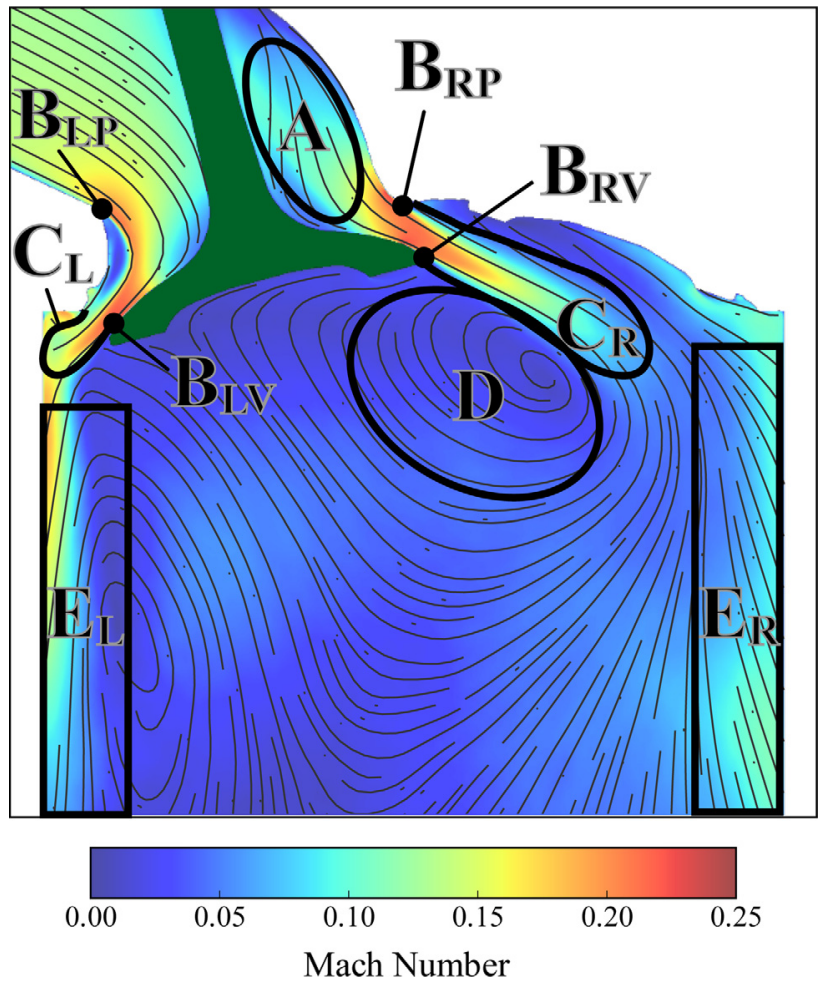

Figure 1

Ensemble-averaged Mach number and streamlines in the valve center plane of a steady-state flow bench configuration. A to E denote regions with characteristic flow phenomena. The subscripts L, R, V and P denote "left", "right", "valve" and "port", respectively.

curvature and shape are determined based on an ad-hoc postprocessing methodology.

\section{METHODOLOGY OF INTAKE JET INVESTIGATION}

This section describes the methodology applied to investigate the intake jet. For this, a characteristic velocity, $v_{\text {char }}$, defined as

$$
v_{\text {char }}=\frac{\dot{m}}{\rho A_{\mathrm{C}}}
$$

is introduced. The intake mass flow and the density are denoted by $\dot{m}$ and $\rho$. The cross-sectional area

$$
A_{\mathrm{C}}=2\left(d_{\mathrm{I}}+g \sin \gamma\right) \pi g
$$

is based on the inner diameter of the valve face $d_{\mathrm{I}}=29 \mathrm{~mm}$ and the seat angle $\gamma=44.6^{\circ}$. The factor 2 represents the two intake valves. The valve gap $g$ is defined as:

$$
g=\cos \gamma h
$$


$h$ : valve lift

$g:$ valve gap

$\gamma: \quad$ valve seat angle

$A_{C}:$ cross sectional area

$d_{A_{C}}:$ mean diameter of $\mathrm{A}_{\mathrm{C}}$

$d_{I}$ : inner valve face diameter

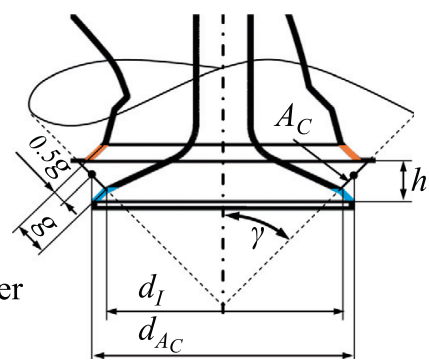

Figure 2

Geometry of the intake valve. Blue and orange lines indicate valve face and intake seat, respectively.

The valve lift is denoted by $h$. Figure 2 illustrates the valve geometry.

Based on these quantities, a valve gap Reynolds number is defined, written as:

$$
R e=\frac{\dot{m} g}{A_{\mathrm{C}} \eta}
$$

with the dynamic viscosity $\eta$.

Figure 3 shows the jet centerline based on an example mean flow bench velocity field. The jet centerline defined by the maximum velocity $[26,27]$ is used for a quantification of the intake jet orientation and its velocity profile in direction of flow.

The reference point for the jet centerline extraction is the outer edge of the valve face in the valve center plane (red dot in Fig. 3). At this point, an evaluation line perpendicular to the valve face is constructed, which is shifted by one valve lift parallel to the valve face in the direction of flow (red vector). The maximum velocity on this evaluation line defines the starting point of the jet centerline $\mathbf{x}_{0}=\mathbf{x}(s=0)$, where $s$ represents a curved coordinate axis (adapted to the jet centerline). The corresponding coordinates can be interpreted as the distance to the starting point along the trajectory. $\mathbf{x}$ is the two-component vector which indicates the position of the jet centerline on the valve center plane parameterized by $s$. Beginning with the starting point, the jet centerline is integrated based on the mean velocity field according to the following equation:

$$
\mathbf{x}(s)=\int_{0}^{\mathbf{s}} \frac{\mathbf{u}\left(\mathbf{x}\left(s^{\prime}\right)\right)}{\left|\mathbf{u}\left(\mathbf{x}\left(s^{\prime}\right)\right)\right|} \mathrm{ds}^{\prime}, \mathbf{x}(s)=\left(\begin{array}{c}
\mathrm{Y}(s) \\
Z(s)
\end{array}\right) \mathbf{u}=\left(\begin{array}{c}
u_{y} \\
u_{z}
\end{array}\right)
$$

The downstream integration limit is $s=30 \mathrm{~mm}$ and the upstream integration limit is $s=-10 \mathrm{~mm}$. This integration was implemented using an integrator from the "SciPy" library [28] with a spatial discretization of $0.1 \mathrm{~mm}$.

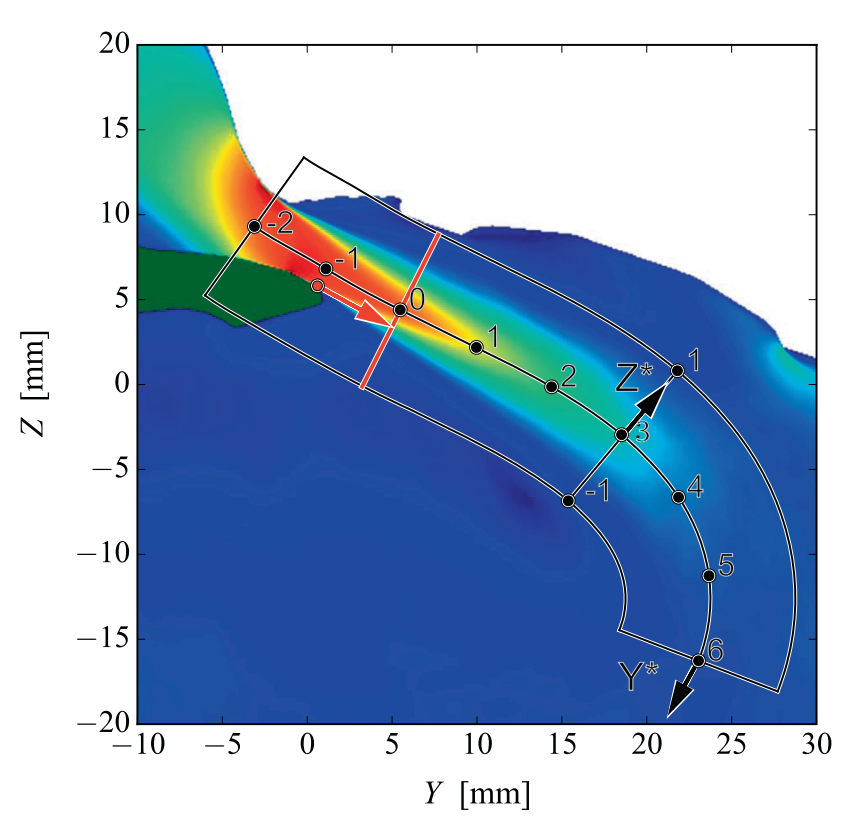

Figure 3

Visualization of the intake jet, the jet centerline and the local $Y^{*}-Z^{*}$ coordinate system. The red dot (reference point), vector and line illustrate the procedure to define the starting point of the jet centerline.

For the following investigations, a trajectory-based $Y^{*}$ $Z^{*}$ coordinate system is introduced. The $Y^{*}-Z^{*}$ plane is the same as the $Y-Z$ plane, while the $X$ axis is the "out-of-plane axis" for both coordinate systems (see right half of Fig. 5 for global coordinate system). $Y^{*}$ represents the dimensionless form of $s$ (normalized by the valve lift). $Z^{*}$ is a dimensionless axis, oriented normal to the trajectory along the $Y^{*}$ axis (i.e. locally adapted). The two enveloping lines are arranged with a distance of one valve lift to the jet centerline and define a sub-area used for further investigations.

\section{EXPERIMENTAL STUDIES}

In the experiment, the engine head was mounted on an optical table, a constant airflow was supplied to the intake at a fixed valve lift, and the resulting flow below one of the intake valves was imaged by PIV. The experimental arrangement is similar to that in a previous work [21], but for completeness a brief overview is given here.

Figure 4 shows the Computer Aided Design (CAD) model of the intake system, the cylinder head, and the outlet tube of the flow bench. In the laboratory frame, the whole assembly lies on its side on an optical table. Nevertheless, throughout this paper the orientation of the cylinder head, valve, and flow is referred as if the head was mounted upright with the valves above the cylinder. The acrylic outlet 


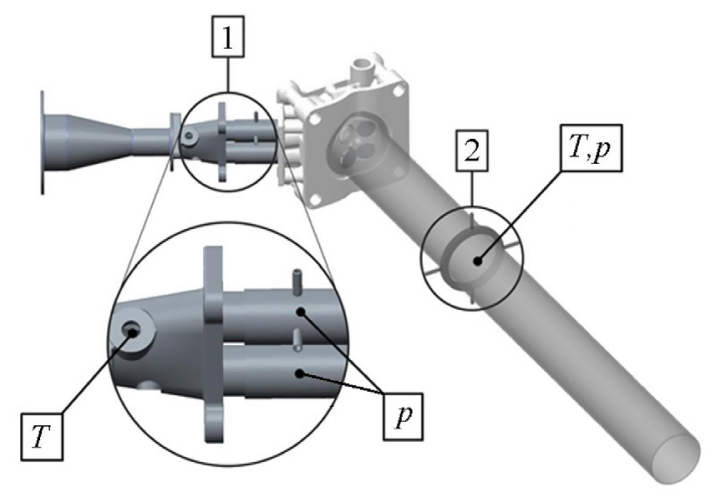

Figure 4

CAD model of the intake port, cylinder head and outlet tube. The model also includes experimental measurement points for temperature $T$ and pressure $p$.

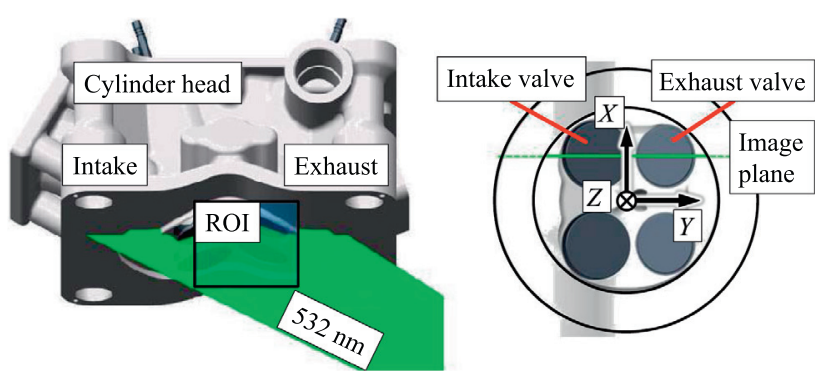

Figure 5

Location of the laser light sheet for PIV measurements. PIV measurements were performed in ROI. Location of the global coordinate system is indicated in the right half. The origin lies in the cylinder head gasket plane.

tube has an inner diameter of $84 \mathrm{~mm}$, corresponding to the bore of the engine. The temperature $T$ and the pressure $p$ were measured in the intake port and the outlet tube. The mass flow upstream of the intake port was calculated from temperature, pressure, and volume flow rate, the latter was measured by a rotary piston meter.

In a plane intersecting the center of one of the intake valves, the two in-plane components of the instantaneous velocity field were measured by PIV (2D-2C PIV). Figure 5 shows the location of the laser light sheet (0.5-0.9 mm thick) and the imaged Region Of Interest (ROI) with respect to the cylinder head. The intake air was seeded with di-ethylhexyl-sebacate droplets generated by a nebulizer [29] ( $\mathrm{LaVi}$ sion). The field of view was illuminated by a double-pulsed Nd:YAG laser (Litron) at $532 \mathrm{~nm}$, and the light scattered by the seeding particles was detected through the acrylic cylinder by a CMOS camera (PCO Edge 5.5, $2560 \times 2160$ pixel). A commercial camera lens projected a field of view of $58 \times 48.9 \mathrm{~mm}^{2}$ onto the detector. The interrogation window size of $32 \times 32$ pixel corresponds to $725 \times 725 \mu \mathrm{m}^{2}$, and a $75 \%$ overlap yielded $4 \times 4$ vectors in each of these interrogation windows. LaVision's DaVis software was used for vector calculation.

The starting point for the subsequent investigations is a series of steady-state PIV measurements with a varying mass flow and valve lift as illustrated in Figure 6. The valve lift was set to 3,5 and $7 \mathrm{~mm}$, while the mass flow was 2, 3 and $4 \mathrm{~kg} \mathrm{~min}{ }^{-1}$. Each flow condition was recorded at a repetition rate of $15 \mathrm{~Hz}$. In addition, some image series at $14 \mathrm{~Hz}$ were acquired. Since both series yielded identical mean flow fields, it seems very unlikely that at either repetition rate the acquisition accidentally coincided with any potentially periodic flow phenomenon. Because of the resulting wide range of velocity magnitudes in the intake jet, a normalized velocity magnitude $|\langle\mathbf{u}\rangle|_{\text {no }}=|\langle\mathbf{u}\rangle| / v_{\text {char }}$ is shown. $v_{\text {char }}$ is the valve-gap velocity (Eq. 1 ), while

$$
\left\langle u_{i}\right\rangle(x)=\frac{1}{N} \sum_{n=1}^{N} u_{i}(x, n)
$$

denotes an ensemble-averaged velocity component based on a total number of $N=300$ samples for this preliminary measurement series. Furthermore, the corresponding valve gap Re number (Eq. 4) is shown.

All operating points result in a similar general flow pattern. A distinctive intake jet $C_{R}$ detaches at the valve face $\mathrm{B}_{\mathrm{RV}}$, penetrates into the combustion chamber, creates a recirculation zone $\mathrm{D}$ and a region of wall reattachment $\mathrm{E}_{\mathrm{R}}$. However, some differences between the flow patterns are discernible for different valve lifts. For $3 \mathrm{~mm}$ and $2 \mathrm{~kg} \mathrm{~min}^{-1}$, the intake jet is bent towards the cylinder head, but becomes directed more downward with an increasing valve lift. A similar effect exists for the mass flow, where the orientation towards the cylinder head at $2 \mathrm{~kg} \mathrm{~min}^{-1}$ is lost more and more for $3 \mathrm{~kg} \mathrm{~min}{ }^{-1}$ and $4 \mathrm{~kg} \mathrm{~min}^{-1}$.

For a quantitative comparison, the velocity components are transformed into the local $Y^{*}-Z^{*}$ coordinate system. This results in a velocity component in the direction of flow $u_{\mathrm{c}}$ (aligned with the $Y^{*}$ axis) and a velocity component across the direction of flow $u_{n}$ (aligned with the $Z^{*}$ axis). Figure 7 shows the normalized ensemble-averaged velocity component in the direction of flow $\left\langle u_{\mathrm{c}}\right\rangle_{\mathrm{no}}=\left\langle u_{\mathrm{c}}\right\rangle / v_{\text {char }}$ along the $Y^{*}$ axis on the jet centerline (Sect. 1) obtained from the measurement series. The intake port is designed to introduce a high level of charge motion (i.e. tumble), which leads to a focussed mass flow in the region of the intake jet $C_{R}$. Due to this, all results exhibit $\left\langle u_{\mathrm{c}}\right\rangle_{\text {no }}$ larger than unity at $Y^{*}=-1$. The exact value depends on $h$ and the valve gap Re number, while combinations of small mass flows and small valve lifts lead to relatively small values for $\left\langle u_{\mathrm{c}}\right\rangle_{\mathrm{no}}$. The highest velocity is obtained by the case with $\dot{m}=4 \mathrm{~kg} \mathrm{~min}^{-1}$ and $h=7 \mathrm{~mm}\left(\right.$ at $\left.Y^{*}=-1\right)$. In previous 

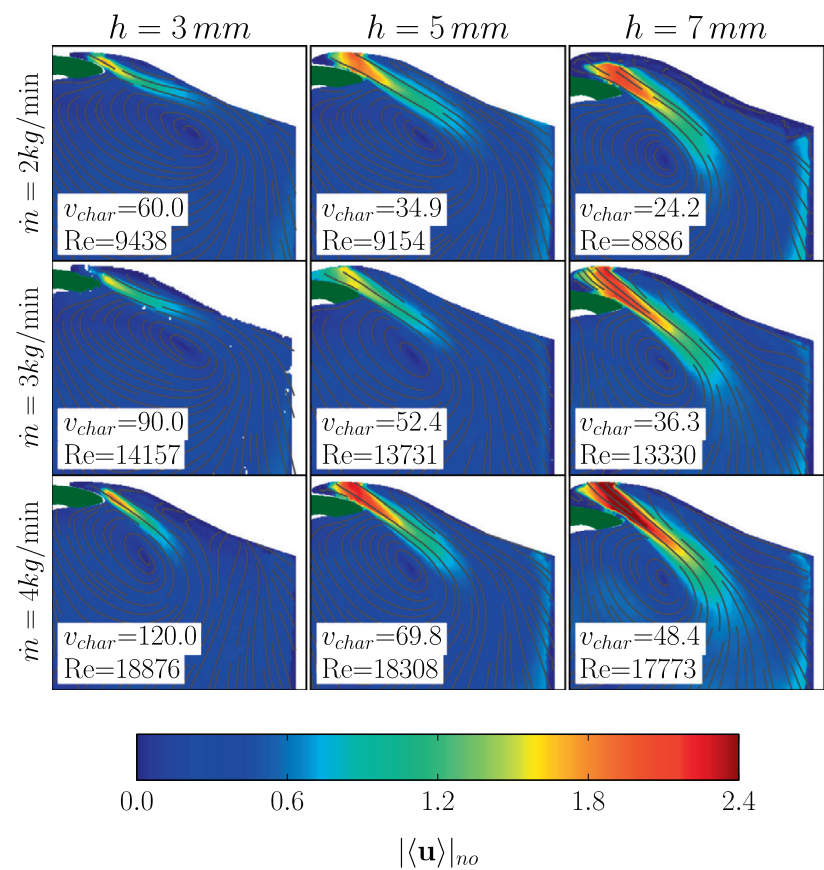

Figure 6

Normalized ensemble-averaged velocity magnitude and streamlines from PIV. Along a row the valve lift $h$ varies, while the valve gap Reynolds number is almost constant. Minor changes in $R e$ within a row result from differences in $A_{\mathrm{C}}$ (Eq. 4).

studies, it was shown that the valve shaft strongly influences the intake jet at the valve center plane $[5,30]$. The differences in $\left\langle u_{\mathrm{c}}\right\rangle_{\text {no }}$ between different cases decrease to $Y^{*} \approx 1.5$ and remain nearly constant afterwards.

For more detailed investigations a valve lift of $h=5 \mathrm{~mm}$ and a mass flow of $\dot{m}=3 \mathrm{~kg} \mathrm{~min}^{-1}$ was chosen for the following reasons:

- mass flow similar to that in unthrottled engine operation at about $2000 \mathrm{rpm}$ (assuming a cylinder displacement of $V_{\mathrm{H}}=0.51$ and boosting to $p_{\mathrm{abs}}=1.5 \mathrm{bar}$ );

- valve lift close to the lift averaged over the open-valve time for typical automotive valve lift profiles;

- minor effects of flow compressibility in the simulation and of particle inertia in the PIV measurements.

\section{NUMERICAL APPROACH}

This section gives an overview of the turbulence models, numerical meshes, boundary conditions and solver.

In this work, the focus is on the results obtained by the Sigma model [31], which is a state-of-the-art Large Eddy Simulation (LES) turbulence model. The eddy viscosity is defined as:

$$
v_{\mathrm{t}}=\left(c_{\mathrm{m}} \Delta\right)^{2} \bar{D}_{m}
$$

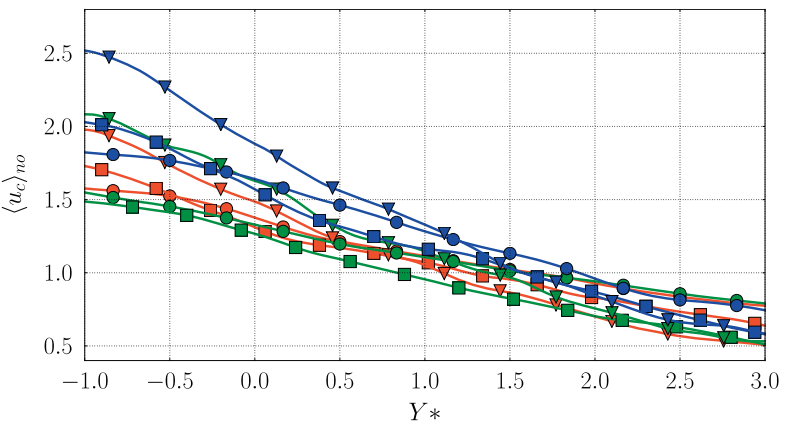

$\bullet 2 \mathrm{~kg} / \mathrm{min} ; 3 \mathrm{~mm} \bullet 3 \mathrm{~kg} / \mathrm{min} ; 3 \mathrm{~mm} \bullet 4 \mathrm{~kg} / \mathrm{min} ; 3 \mathrm{~mm}$ $\square \square 2 \mathrm{~kg} / \mathrm{min} ; 5 \mathrm{~mm} \square \square 3 \mathrm{~kg} / \mathrm{min} ; 5 \mathrm{~mm} \square 4 \mathrm{~kg} / \mathrm{min} ; 5 \mathrm{~mm}$ $\longmapsto 2 \mathrm{~kg} / \mathrm{min} ; 7 \mathrm{~mm} \gg 3 \mathrm{~kg} / \mathrm{min} ; 7 \mathrm{~mm} \gg 4 \mathrm{~kg} / \mathrm{min} ; 7 \mathrm{~mm}$

Figure 7

Normalized velocity in the direction of flow on the jet centerline along dimensionless distance $\left(Y^{*}\right)$ to starting point.

with the model-specific constant $c_{\mathrm{m}}=1.5$ [32] and the local grid size $\Delta$. The differential operator $\bar{D}_{\mathrm{m}}$ is defined as:

$$
\bar{D}_{\mathrm{m}}=\frac{\sigma_{3}\left(\sigma_{1}-\sigma_{2}\right)\left(\sigma_{2}-\sigma_{3}\right)}{\sigma_{1}^{2}}
$$

with the singular values $\sigma_{i}$ of the matrix $G_{i j}=\partial \bar{u}_{k} / \partial x_{i} \partial \bar{u}_{k} / \partial x_{j}$. $\bar{\Phi}$ indicates a filtered (LES) or averaged (Unsteady Reynolds Averaged Navier-Stokes (URANS)) quantity, with the overbar omitted in the following for simplicity. An important feature of this model is that it exhibits the correct scaling for the eddy viscosity $v_{\mathrm{t}}=\mathcal{O}\left(y^{3}\right)$ close to the wall $(y \rightarrow 0)$. Furthermore, it has the capability to capture the laminar-to-turbulent transition due to zero eddy viscosity in the case of pure shear [31].

Another turbulence model investigated here is the DESSST model [33], which was successfully applied to IC engine flows in $[4,21,34]$ and is based on the averaged form of the Navier-Stokes equations. It belongs to the group of hybrid URANS/LES turbulence models (originally suggested in [35]), which revert to a URANS formulation when the local grid size is not sufficient for LES. The eddy viscosity is calculated as:

$$
v_{\mathrm{t}}=\frac{a_{1} k}{\max \left(a_{1} \omega, S F_{2}\right)}
$$

with the turbulent kinetic energy $k$, the turbulent frequency $\omega$ [36] and $S=\frac{1}{2}\left(\frac{\partial u_{i}}{\partial x_{j}} \frac{\partial u_{j}}{\partial x_{i}}\right) \cdot a_{1}$ and $F_{2}$ denote a constant and a blending function [37]. To obtain the hybrid behavior, the dissipation term of the $k$-equation of the underlying SST URANS approach [37] is modified as:

$$
\varepsilon_{\mathrm{SST}}=\beta^{*} k \omega=\frac{k^{\frac{3}{2}}}{L_{\mathrm{t}}} \rightarrow \varepsilon_{\mathrm{DES}}=\frac{k^{\frac{3}{2}}}{L_{\mathrm{DES}}}
$$


with the constant $\beta^{*}$. If there is a sufficiently fine spatial resolution, the model-specific length scale

$$
L_{\mathrm{DES}}=\min \left(L_{\mathrm{t}}, c_{\mathrm{DES}} \Delta\right)
$$

switches from the integral length scale $L_{\mathrm{t}}$ (i.e. URANS approach) to a grid-dependent length scale $c_{\mathrm{DES}} \Delta$ (i.e. LES approach). An advantage of the hybrid models is that the reversion to a URANS formulation can lead to a significant decrease in computational effort, especially for wall-bounded flows [38]. A disadvantage of these models is the so-called "grey area", which denotes the region where the transition from modeled to resolved turbulent structures takes place. For IC engine flows, it typically affects the region close to the valve shaft and the valve gap. This transition has to be captured correctly for reliable results (discussed in Sect. 4.2).

Figure 8 illustrates two of the meshes investigated in this work, namely the coarse and fine meshes, which are identical in their topology. The numerical domain is based on the CAD model shown in Figure 4. Body conformal and hybrid meshes consisting of tetrahedral, prism and hexahedral elements are used. Mesh refinements are located in the region of the intake jet $C_{L}$ and $C_{R}$ to capture the strong gradients in this region. Further refinements are placed at the separation edge $B_{L P}$ and in the vicinity of the valve shaft (A) to resolve small-scale fluctuations generated directly upstream of the valve gap. Prism layers are used to obtain an improved resolution of the boundary layer in an attempt to place the first layer within the viscous sublayer (i.e. $\left.y^{+}<5\right)[1,5,39]$. In addition, a medium mesh is investigated, which is not shown for the sake of brevity. Table 1 summarizes the mesh parameters.

In the following, only specific combinations of turbulence models and meshes are analyzed. These combinations and their abbreviations are:

- DES $\rightarrow$ DES-SST on medium mesh;

- SC $\rightarrow$ Sigma on Coarse mesh;

- SM $\rightarrow$ Sigma on Medium mesh;

- SF $\rightarrow$ Sigma on Fine mesh.

Corresponding to the previously specified operating point, the simulations were performed at a temperature of $298 \mathrm{~K}$. For the inlet boundary condition, a mass flow of $3 \mathrm{~kg} \mathrm{~min}{ }^{-1}$ (Sect. 2) with resolved turbulent fluctuations was generated by a separate turbulent pipe-flow simulation. The valve lift was set to $h=5 \mathrm{~mm}$. For the outlet boundary conditions, ambient pressure was used. All numerical investigations were run for a wall-clock time of $0.06 \mathrm{~s}$. Based on the time step in Table 1, a Courant-Friedrichs-Lewy number smaller than unity $(\mathrm{CFL}<1)$ is obtained for the entire domain. Previous numerical studies [21] have shown that the incompressible continuity and momentum equations are suitable for this case. This is also confirmed by the results obtained here. Figure 1 shows the Mach number based on
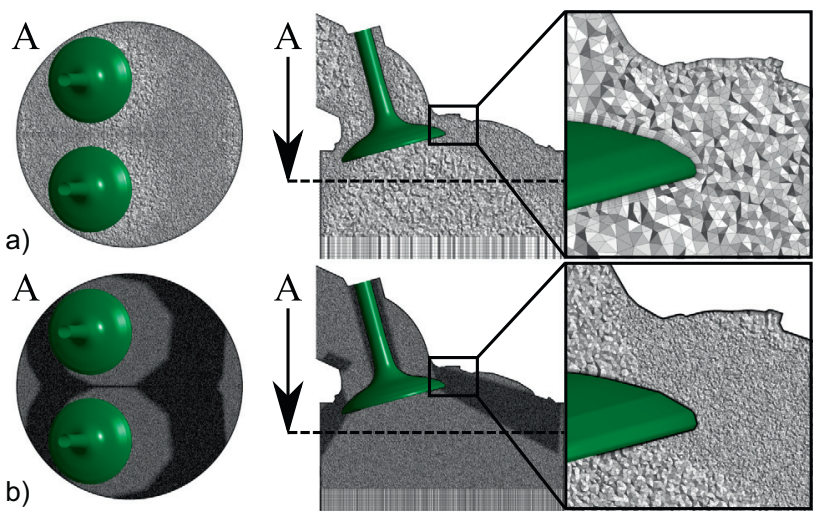

Figure 8

Three different meshes are investigated (Tab. 1), though only the coarse mesh a) and the fine mesh b) are shown for the sake of brevity. Left: top view of the mesh $5 \mathrm{~mm}$ below the cylinder head gasket position. Middle/Right: mesh topology in the valve center plane.

TABLE 1

Parameters for the selected meshes. $\Delta_{x}$ denotes the element width.

\begin{tabular}{c|c|c|c}
\hline Mesh & Coarse & Medium & Fine \\
\hline Grid points & $5.7 \times 10^{6}$ & $25.1 \times 10^{6}$ & $155.1 \times 10^{6}$ \\
\hline Elements & $15.3 \times 10^{6}$ & $74.4 \times 10^{6}$ & $493.4 \times 10^{6}$ \\
\hline Wall prisms & 10 & 15 & 20 \\
\hline$\Delta_{x \text { chamber }}$ & $1.0 \mathrm{~mm}$ & $0.5 \mathrm{~mm}$ & $0.25 \mathrm{~mm}$ \\
\hline$\Delta_{\text {xrefined }}$ & $0.5 \mathrm{~mm}$ & $0.25 \mathrm{~mm}$ & $0.125 \mathrm{~mm}$ \\
\hline$\Delta t$ & $6 \mu \mathrm{s}$ & $3 \mu \mathrm{s}$ & $1.5 \mu \mathrm{s}$ \\
\hline CPU & 144 & 288 & 3000 \\
\hline CPUh & $1 \times 10^{4}$ & $7 \times 10^{4}$ & $1 \times 10^{4}$ \\
\hline
\end{tabular}

the ensemble-averaged velocity magnitude, which is less than 0.25 over the entire valve center plane.

All simulations were performed using ANSYS CFX Release 16.0. The transport equations are discretized using a node-centered finite volume method, which is conservative and time-implicit [40-42]. A control volume is created around each node of the mesh. The fluxes are computed at the integration points located at the sub faces between two control volumes. Pressure-velocity coupling is achieved via the algorithm developed in [43]. The discrete system of equations is solved by a coupled algebraic multi-grid method [42]. A central differencing scheme in space and a second-order backward scheme in time are used for the simulations to minimize numerical diffusion. Previous studies showed the suitability of the solver for SRS in IC engines [4, 5, 18, 21, 34, 44]. On Intel Xeon E5-2680v3 processors, 
an overall computing time of $1.08 \times 10^{6} \mathrm{CPUh}$ was needed for all the simulations presented.

\section{INVESTIGATION OF THE INTAKE FLOW}

In this section, the flow field based on the horizontal and vertical component on the valve center plane is analyzed, with a focus on the intake jet in the vicinity of the intake valve. First, an overview of the general flow topology based on the instantaneous velocity magnitude is given. Then, the ensemble-averaged velocity and the Root Mean Square (RMS) of the velocity fluctuations in experiment and simulation are compared qualitatively and quantitatively. Finally, the intake jet is investigated in terms of its orientation and penetration depth. The investigations are limited to the case with $\dot{m}=3 \mathrm{~kg} \mathrm{~min}^{-1}$ and $h=5 \mathrm{~mm}$. All statistic quantities presented in the following are based on a total number of $N=600$ samples for the experiment and $N=400$ samples for each simulation.

\subsection{General Flow Structure}

Figure 9 illustrates the instantaneous velocity magnitude from SF and PIV. Starting at the intake, a significant amount of resolved small-scale structures occur at the separation edge and at the valve shaft, represented by region $\mathrm{A}$ and $\mathrm{B}_{\mathrm{LP}}$, respectively. These structures are transported into the combustion chamber by the mean flow, which can be seen by the scattered spots in the velocity magnitude within the valve gap. This transport through the valve gap was also mentioned in [21] based on a frequency analysis. At $\mathrm{C}_{\mathrm{L}}$, the intake jet (outside of the experimental ROI) detaches at the valve face $\mathrm{B}_{\mathrm{LV}}$ and is deflected downward by the cylinder liner $\left(\mathrm{E}_{\mathrm{L}}\right)$. The intake jet at $C_{R}$, identifiable in both the experiment and the simulation, detaches at $\mathrm{B}_{\mathrm{RV}}$ and $\mathrm{B}_{\mathrm{RP}}$ and penetrates into the combustion chamber towards the center. This part of the jet is the main contributor to the recirculation zone (D) and is characterized by significant variation in its position and shape. A rough idea of this variability can be obtained from the illustrated time steps. Due to the high velocity gradient between the intake jet and the surrounding fluid, a significant number of turbulent structures are produced in the corresponding transition zone. Further downstream, the jet is deflected by the cylinder liner $\left(E_{R}\right)$ towards the outlet. Based on this visual comparison, experiment and SF are in good agreement in terms of the general flow structure, jet dynamics and level of resolved small-scale structures.

\subsection{Qualitative and Quantitative Analysis of the Flow Field}

Figure 10 shows an instantaneous field of the velocity magnitude and the ensemble-averaged fields from PIV, DES, SC,

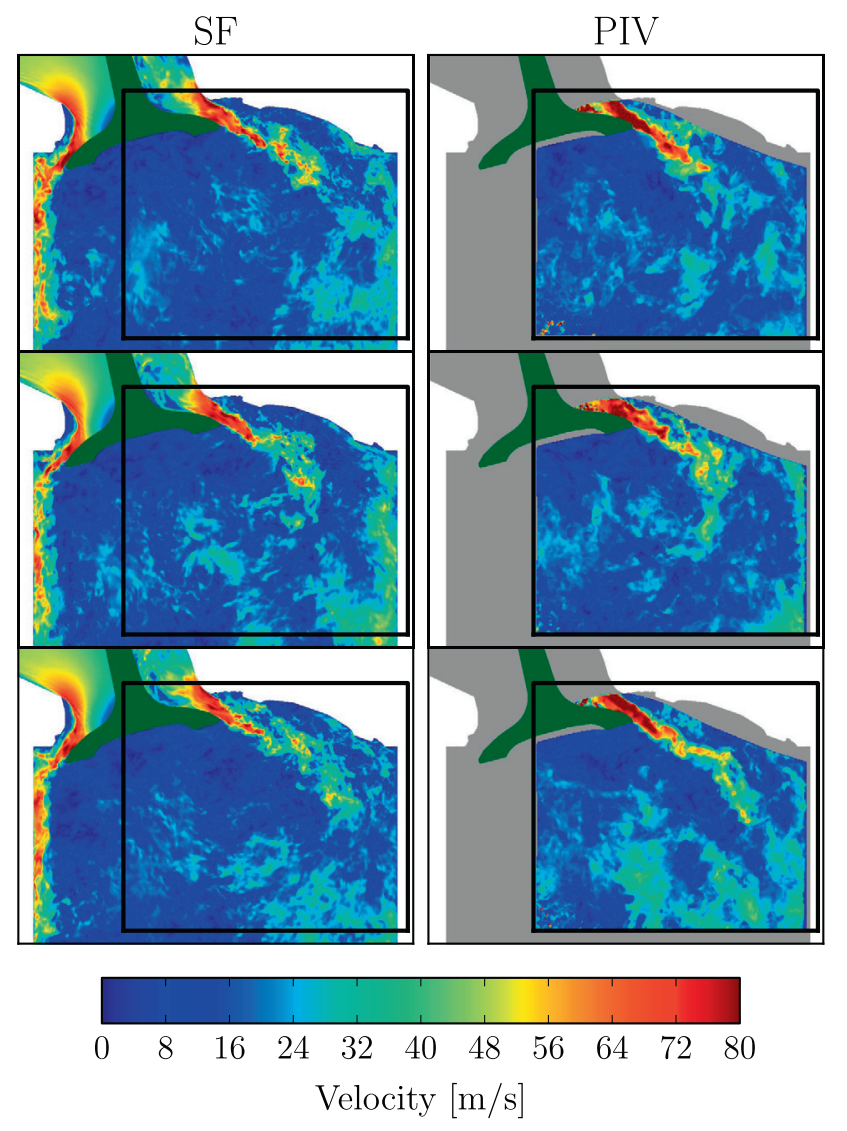

Figure 9

Instantaneous velocity magnitude at arbitrary time steps from $\mathrm{SF}$ and PIV on the valve center plane. Black frame for the ROI from experiment

SM and SF. The previously described flow topology is captured very well by all simulations. For SF and SM computations, turbulent structures are generated directly at the separation edges of the valve $\left(\mathrm{B}_{\mathrm{LV}}\right.$ and $\left.\mathrm{B}_{\mathrm{RV}}\right)$. In contrast, for the DES model, the turbulent structures can be observed considerably further downstream. This suggests that the region in the first part of the jet is still influenced by the URANS approach in the boundary layer on the valve/port surface. However, further downstream, the resolved turbulent structures in the intake jet region are similar to the ones of the Sigma model. As expected, the amount of resolved turbulent structures obtained by the Sigma model increases with an increasing number of grid points. Based on visual inspection of the resolved structures, the DES lies in between SC and SM, while SF and PIV are close together. The following observations are made for an increasing number of grid points for the Sigma model:

- increasing number of resolved small-scale structures of the flow downstream of the separation edge $B_{L P}$;

- more pronounced jet breakup close to the separation edge of the valve $\left(\mathrm{B}_{\mathrm{LV}}\right.$ and $\left.\mathrm{B}_{\mathrm{LV}}\right)$. 


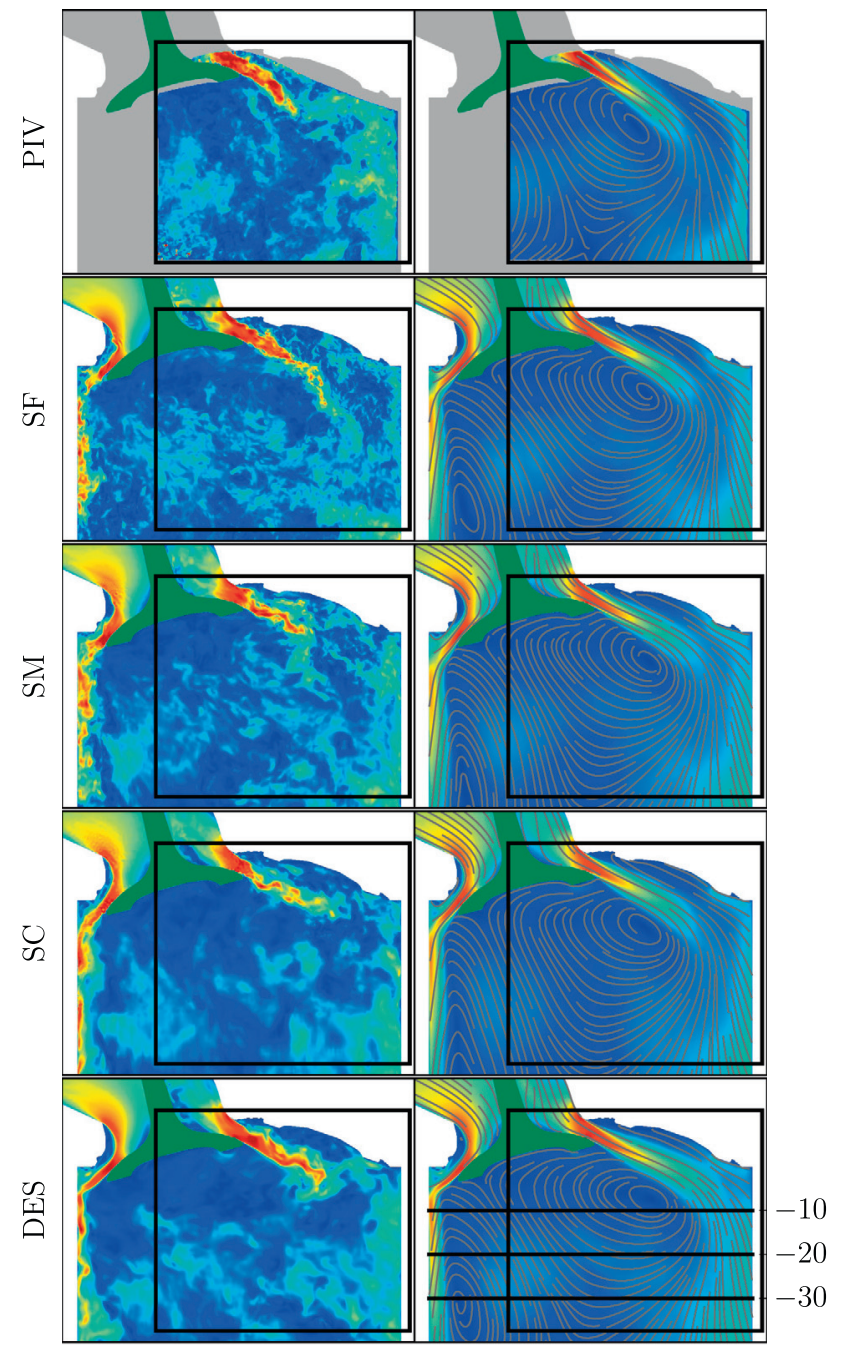

a)

b)

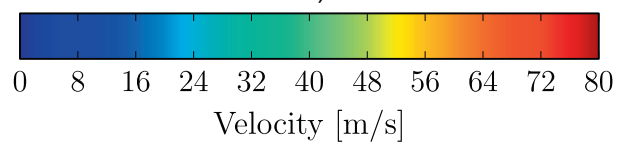

Figure 10

Instantaneous a) and ensemble-averaged b) velocity magnitude obtained by PIV, DES-SST (DES), SC, SM and SF in the valve center plane. Lines at $Z=-10 \mathrm{~mm},-20 \mathrm{~mm}$ and $-30 \mathrm{~mm}$ indicate axial positions for the quantitative comparison shown in Figure 11

In the ensemble-averaged velocity magnitude, only small differences can be identified. With the exception of slight deviations in region $\mathrm{D}, \mathrm{SC}, \mathrm{SM}$ and SF are almost identical. There is also good agreement with the PIV, which exhibits a slightly higher velocity magnitude within the valve gap. The largest deviations exist in the region $C_{R}$. Compared to the simulation results, the intake jet obtained by the PIV exhibits a stronger curvature and is bent more towards the combustion chamber. These deviations become particularly obvious when the PIV measurements and the DES model are compared, since the DES results exhibit the most pronounced orientation towards the cylinder head. Close to the corresponding reattachment region $\mathrm{E}_{\mathrm{R}}$, the flow obtained by the DES model differs from those predicted by the other methods by a slightly higher velocity magnitude, which results from the increased intake jet penetration depth (compared to the other models).

Figure 11 illustrates the ensemble-averaged velocity components $\left\langle u_{z}\right\rangle$ and $\left\langle u_{y}\right\rangle$ on different vertical positions $(Z=-10 \mathrm{~mm},-20 \mathrm{~mm}$ and $-30 \mathrm{~mm}$; lines indicated in Fig. 10). First, the zone $E_{R}$ is considered, where the intake jet reattaches at the cylinder. Here, the simulation results are close together for all $Z$ locations, while there are small deviations to the PIV in the vicinity of the wall. At $Z=-10 \mathrm{~mm}$, the footprint of the intake jet $\mathrm{C}_{\mathrm{R}}$ can be identified in the range between $20 \mathrm{~mm}<Y<33 \mathrm{~mm}$. Consistent with the previous observations, the profiles for $\left\langle u_{z}\right\rangle$ obtained by PIV and DES indicate a stronger orientation of the intake jet towards the center of the cylinder and the cylinder head. The recirculation zone D is located at the center of the cylinder, clearly identifiable at $Z=-20$ and $-30 \mathrm{~mm}$ by the moderate slope in the $\left\langle u_{z}\right\rangle$ profile. This slope represents the flow towards the cylinder head (positive $\left\langle u_{z}\right\rangle$ ), the region aligned to the center of rotation (zero crossing) and the flow towards the outlet (negative $\left\langle u_{z}\right\rangle$ ). Comparing simulation and experimental results in region $\mathrm{D}$, a good general agreement can be observed, while there are small differences at specified regions. For example, at $Z=-20$ and $-30 \mathrm{~mm},\left\langle u_{z}\right\rangle$ obtained in the SC case differs from the other simulation results in the range between $-33 \mathrm{~mm}<$ $Y<-20 \mathrm{~mm}$. Considering the horizontal velocity component $\left\langle u_{y}\right\rangle$, shown as dashed lines, all the simulation results are very similar. There are deviations to the PIV, especially at $Z=-30 \mathrm{~mm}$. Finally, the region $\mathrm{E}_{\mathrm{L}}$ is considered, where the intake jet $C_{L}$ reattaches to the wall. Here, all the simulation results exhibit almost identical values for $\left\langle u_{z}\right\rangle$ and $\left\langle u_{y}\right\rangle$ (no PIV data available). At $Z=-10 \mathrm{~mm}$, a vertical velocity of $\left\langle u_{z}\right\rangle \approx-60 \mathrm{~ms}^{-1}$ is obtained, which changes to $\left\langle u_{z}\right\rangle \approx-48 \mathrm{~ms}^{-1}$ at $Z=-30 \mathrm{~mm}$. Corresponding to this decrease in velocity magnitude, the wall-bounded flow notably widens from $Z=-10 \mathrm{~mm}$ to $Z=-30 \mathrm{~mm}$, identifiable by the decreasing velocity gradient.

The RMS of the resolved in-plane velocity fluctuations is calculated as:

$$
\mathrm{RMS}=\sqrt{\frac{1}{2}\left(\left\langle u_{y}^{\prime 2}\right\rangle+\left\langle u_{z}^{\prime 2}\right\rangle\right)}
$$

The fluctuations are calculated based on the Reynolds decomposition $u_{i}^{\prime}=u_{i}-\left\langle u_{i}\right\rangle$. As shown in Figure 12, there is a significant increase in resolved fluctuations at the separation edge and behind the valve shaft $\left(\mathrm{B}_{\mathrm{LP}}\right.$ and $\mathrm{A}$; both outside of the experimental ROI), which is consistent with 

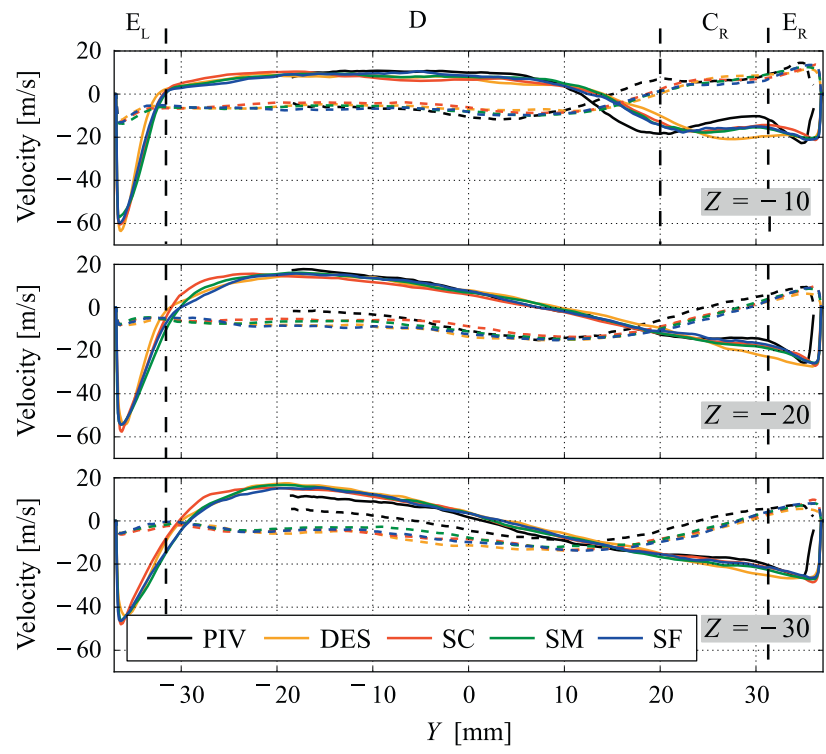

Figure 11

Ensemble-averaged vertical velocity $\left\langle u_{z}\right\rangle(-)$ and horizontal velocity $\left\langle u_{z}\right\rangle(--)$ obtained by PIV, DES-SST (DES), SC, $\mathrm{SM}$ and $\mathrm{SF}$ on lines at $Z=-10 \mathrm{~mm},-20 \mathrm{~mm}$ and $-30 \mathrm{~mm}$. The different phenomena (Fig. 1) are indicated by vertical lines. Please note that the intake jet $C_{R}$ is limited to $Z=-10 \mathrm{~mm}$.

observations in [16]. A further increase occurs at the separation edges of the valve seat/face $\left(B_{R P}, B_{L V}\right.$ and $\left.B_{R V}\right)$, and the highest level of fluctuations is obtained in the intake jet $C_{R}$. These fluctuations decay downstream towards the outlet. Compared to the simulation, the PIV exhibits higher RMS in the intake jet region $\mathrm{C}_{\mathrm{R}}\left(\mathrm{RMS}>30 \mathrm{~ms}^{-1}\right)$ and in the center of the cylinder $\left(\mathrm{RMS} \approx 15 \mathrm{~ms}^{-1}\right.$ ). Also, there are high RMS values in the bottom left corner of the ROI and upstream of the valve gap next to the intake valve. These two "hot spots" can be traced back to an inaccuracy in the measurements [16] and will be neglected in the following. Comparing DES and SC, similar results are obtained within the intake port $\left(A\right.$ and $\left.B_{L P}\right)$ and the intake jet $C_{L}$. In the region $C_{R}$ of the DES, the bulk of the high RMS region is slightly shifted towards the cylinder head, which is consistent with the shift identified in the ensemble-averaged velocities. As discussed above, the SF exhibits higher fluctuations than the other simulations, which is particularly identifiable behind the valve shaft (A) and downstream of the separation edges $B_{L P}, B_{R P}, B_{L V}$ and $B_{R V}$. In the center of the cylinder, the DES and the SF show a similar level of velocity fluctuations, while the lowest values are obtained by SC. The results for SM are in between SF and SC and are not shown for the sake of brevity.

A quantitative comparison of the in-plane velocity fluctuations obtained by experiment and simulation at different vertical positions is shown in Figure 13. The highest



Figure 12

RMS of in-plane velocity fluctuations obtained by PIV, DESSST (DES), SC, SM and SF in the valve center plane. Lines at $Z=-10 \mathrm{~mm},-20 \mathrm{~mm}$ and $-30 \mathrm{~mm}$ indicate vertical positions for a quantitative comparison.

fluctuations occur within the shear layer of the reattachment zone $\mathrm{E}_{\mathrm{L}}\left(\mathrm{RMS} \approx 18 \mathrm{~ms}^{-1}\right.$ at about $Y=-33 \mathrm{~mm}$ ), obtained by SM and SF. Within the recirculation zone $\mathrm{D}$, there is a decrease in RMS to a global minimum, with its exact $Y$-value depending on the vertical position. From this global minimum to $Y \approx 0 \mathrm{~mm}$, there is an increase in velocity fluctuations followed by a slight decrease towards the other side of the cylinder liner for $Z=-20 \mathrm{~mm}$ and $-30 \mathrm{~mm}$. In contrast, there is a significant increase in the RMS values between $Y=10 \mathrm{~mm}$ and $Y=20 \mathrm{~mm}$ at $Z=-10 \mathrm{~mm}$, which results from the intake jet $C_{R}$. Corresponding with Figure 12, PIV exhibits higher velocity fluctuations (compared to simulation) at $C_{R}$ and in the center of the cylinder. The high RMS values at $Z=-30 \mathrm{~mm}$ and $Y \approx-18 \mathrm{~mm}$ correspond to experimental artifacts that can also be seen in Figures 10 and 12 (insufficient laser illumination). The SF and the SM are very similar in most regions, while the SC and the DES exhibit the lowest velocity fluctuations.

\subsection{Investigation of the Jet Position and Penetration Depth}

The following section investigates the orientation, curvature, velocity profiles and penetration depth of the ensembleaveraged intake jet along the jet centerline obtained by PIV and simulation at the valve center plane. 



Figure 13

RMS of in-plane velocity fluctuations obtained by PIV, DES$\mathrm{SST}$ (DES), SC, SM and SF on lines at $Z=-10 \mathrm{~mm}$, $-20 \mathrm{~mm}$ and $-30 \mathrm{~mm}$ (indicated in Fig. 12). The different phenomena (Fig. 1) are indicated by vertical lines. Please note that the intake jet $C_{\mathrm{R}}$ is limited to the $Z=-10 \mathrm{~mm}$ location.

Figure 14 illustrates the jet centerlines obtained by PIV, DES, SC, SM and SF, extracted by the method described in Section 1. Within the valve gap, the jet centerlines are close together and almost aligned with the valve face. Only small differences between PIV and the simulation are identified in this region. Leaving the valve gap, the PIV exhibits a slight deflection towards the center of the cylinder, while the simulation results are curved towards the cylinder head. Apart from this, the initial intake jet is less influenced by the in-cylinder flow field, identifiable by the almost straight jet centerlines. Due to the method reconstructing the jet centerline, small differences close to the valve gap lead to strong deviations in the intake jet position further downstream $\left(Y^{*}=2\right)$. Beginning with $Y^{*} \approx 3$, strong curvature occurs, while there are notable differences between the simulation results. The jet centerline of the DES model shows the smallest curvature, and the Sigma model shows increasing curvature with increasing spatial resolution. The intake jet obtained by SF agrees best with the PIV measurements in terms of its position, although there are still non-negligible differences between the two results.

For a more quantitative comparison of the jet centerlines, their curvature $\kappa$ in the valve center plane, defined as:

$$
\kappa=\frac{Z^{\prime} Y^{\prime \prime}-Z^{\prime \prime} Y^{\prime}}{\left(z^{\prime 2}+y^{\prime 2}\right)^{3 / 2}}
$$

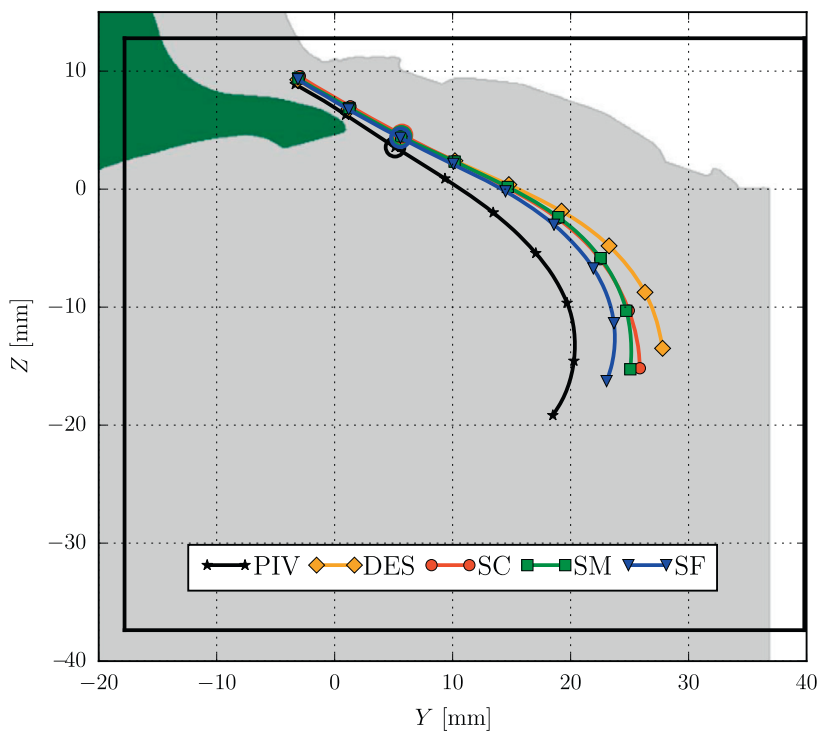

Figure 14

Jet centerline obtained by simulations and PIV based on the ensemble-averaged velocity field in the valve center plane. The starting point $\left(Y^{*}=0\right)$ on each jet centerline is denoted by a circle. The symbols (e.g. squares) are distributed in intervals of $\Delta Y^{*}=1$. The black frame indicates the experimental ROI.

is considered next [45]. For the evaluation of the second derivative, the jet centerline was fitted to cubic splines [46] using the "SciPy" library [28].

The result of this procedure is presented in Figure 15. There are only small differences between the PIV and the simulations in the range of $Y^{*}=-2$ to $Y^{*}=0$. Within the valve gap (up to $Y^{*}=-1$ ), the PIV shows a negative curvature (towards the center of combustion chamber) and the simulation results show zero or slightly positive curvature (towards the cylinder head). Good agreement is identified between $Y^{*}=0$ and $Y^{*}=3$, where all results show a small positive curvature. Larger differences exist from $Y^{*}=3$ to the "end" of the jet centerline at $Y^{*}=6$, and the PIV and SF exhibit a similar trend. Starting from $Y^{*}=3$, there is an increase in curvature to a value of $\kappa=-317 \mathrm{~m}^{-1}$ at $Y^{*}=4.48(\mathrm{PIV})$ and $\kappa=-403.6 \mathrm{~m}^{-1}$ at $Y^{*}=5.16(\mathrm{SF})$, followed by a steady decrease towards $Y^{*}=6$. The same general trend is obtained by the DES model, while it exhibits significantly smaller values than the other results. In contrast, SC and SM exhibit a steady increase in curvature up to $Y^{*}=6$.

The normalized ensemble-averaged velocity in the direction of the flow along the jet centerline is plotted in Figure 16. In these velocity profiles three zones are defined.

Zone I starts upstream of the starting point $\left(Y^{*}=0\right)$ within the intake port and corresponds to the region where the fluid is accelerated. RMS values are low and nearly constant. 


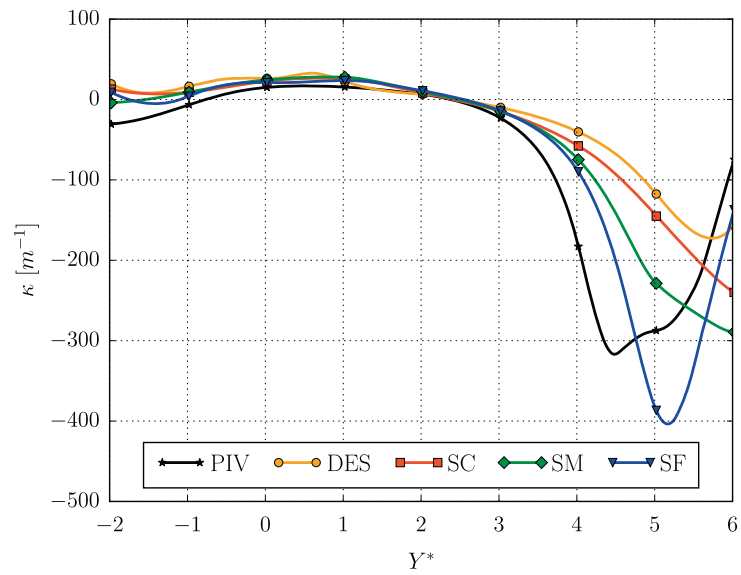

Figure 15

Curvature of the jet centerline along the dimensionless distance from the starting point $\left(Y^{*}=0\right)$ obtained by PIV, DES-SST (DES), SC, SM and SF.

For all results, the maximum velocity, which marks the end of Zone I, is located at $Y^{*}=-1.3$ corresponding to the region with the smallest effective cross-sectional area.

Zone II is defined by a moderate (and almost linear) velocity decrease and increasing RMS until $\left\langle u_{c}\right\rangle_{\mathrm{no}}=1.2$. The exact location for $\left\langle u_{c}\right\rangle_{\text {no }}=1.2$ varies for each result around $Y^{*}=0$ (grey area in Fig. 16). Zone II represents some kind of potential core of a round or planar turbulent jet. Typically, the potential core is defined by the region in which the centerline velocity remains constant and equal to the exit velocity of the jet $[47,48]$. The axis symmetry of the intake valve results in a circumferential expansion of the crosssectional area. Due to this, an immediate velocity decrease on the jet centerline is expected downstream of the acceleration zone (i.e. Zone I).

Zone III marks the far field of the intake jet, which can be identified by the stronger velocity decrease and the moderate decrease of RMS on the jet centerline.

In general, similar trends are obtained in all results and the different zones are clearly identifiable. SC and SM are almost identical, while SF predicts a slightly increased velocity in the direction of flow in Zone I and Zone II. Starting at $Y^{*}=-2$, the DES model shows marginal higher values for $\left\langle u_{c}\right\rangle_{\text {no }}$ than the Sigma model (independent of the grid). These differences increase continuously along the jet centerline. Compared to the results of the Sigma model, the PIV shows a significantly higher $\left\langle u_{c}\right\rangle_{\text {no }}$ in Zone I and Zone II, while these differences vanish at the beginning of Zone III. Regarding the normalized RMS values in the direction of flow, the highest values are obtained by the PIV exhibits, while the DES shows the lowest RMS values in Zone I and Zone II. The results of SC, SM and SF are almost identical. In Zone III, the velocity and the RMS values from PIV and



Figure 16

Normalized velocity $(-)$ and RMS $(---)$ in the direction of flow along the jet centerline $\left(Y^{*}\right)$ obtained by PIV, DES-SST (DES), SC, SM and SF.

the Sigma model are close together, which is particularly true for SF.

Figure 17 shows $\left\langle u_{c}\right\rangle_{\text {no }}$ in the transformed $Y^{*}$ - $Z^{*}$ plane within the sub-area (Fig. 3). Iso-contours are shown at $\left\langle u_{c}\right\rangle_{\text {no }}=1.2,0.8,0.6$ and 0.4 . Vertical lines indicate the boundaries between the different zones with regard to the jet centerline. The end of Zone I, defined by the maximum velocity on the jet centerline is located at $Y^{*}=-1.3$ for all results. In contrast to Figure 16, here, $\left\langle u_{c}\right\rangle_{\mathrm{no}}=1.2$ is used to mark the borderline between Zone II and Zone III. Its location differs from model to model. As discussed above, the DES predicts the largest penetration depth (measured by the locations of the velocity iso-contours) and the PIV shows an increased velocity within the valve gap (i.e. in Zone I). Except for the DES model, all models exhibit similar results in terms of the penetration depth, spreading rate and general topology of the intake jet. In the results obtained by the Sigma model, the $\left\langle u_{c}\right\rangle_{\text {no }}=1.2$ contour is located further downstream as the spatial resolution increases. In contrast, the penetration depth in terms of $\left\langle u_{c}\right\rangle_{\text {no }}=0.4$ is further upstream towards the valve gap for finer meshes.

Figure 18 shows $\left\langle u_{c}\right\rangle_{\text {no }}$ along the jet cross-section $\left(Z^{*}\right.$ axis) at $Y^{*}=-1,0$ and 1 obtained by PIV, DES, SC, $\mathrm{SM}$ and SF. The velocity profiles at $Y^{*}=-1$ show an asymmetric top-hat-like profile with a strong gradient at negative $Z^{*}$ values (valve side). As discussed in Section 1 , this location corresponds to the outer edge of the valve face $\left(\mathrm{B}_{\mathrm{RV}}\right)$, where the intake jet detaches. The high velocity gradient results from a wall-bounded flow upstream of this separation edge $\left(Y^{*}<-1\right)$. On the intake port side (positive $Z^{*}$ values), the gradient is not that high. Here, the separation at the intake seat $\mathrm{B}_{\mathrm{RP}}\left(Y^{*} \approx-2\right)$ leads to a spreading 

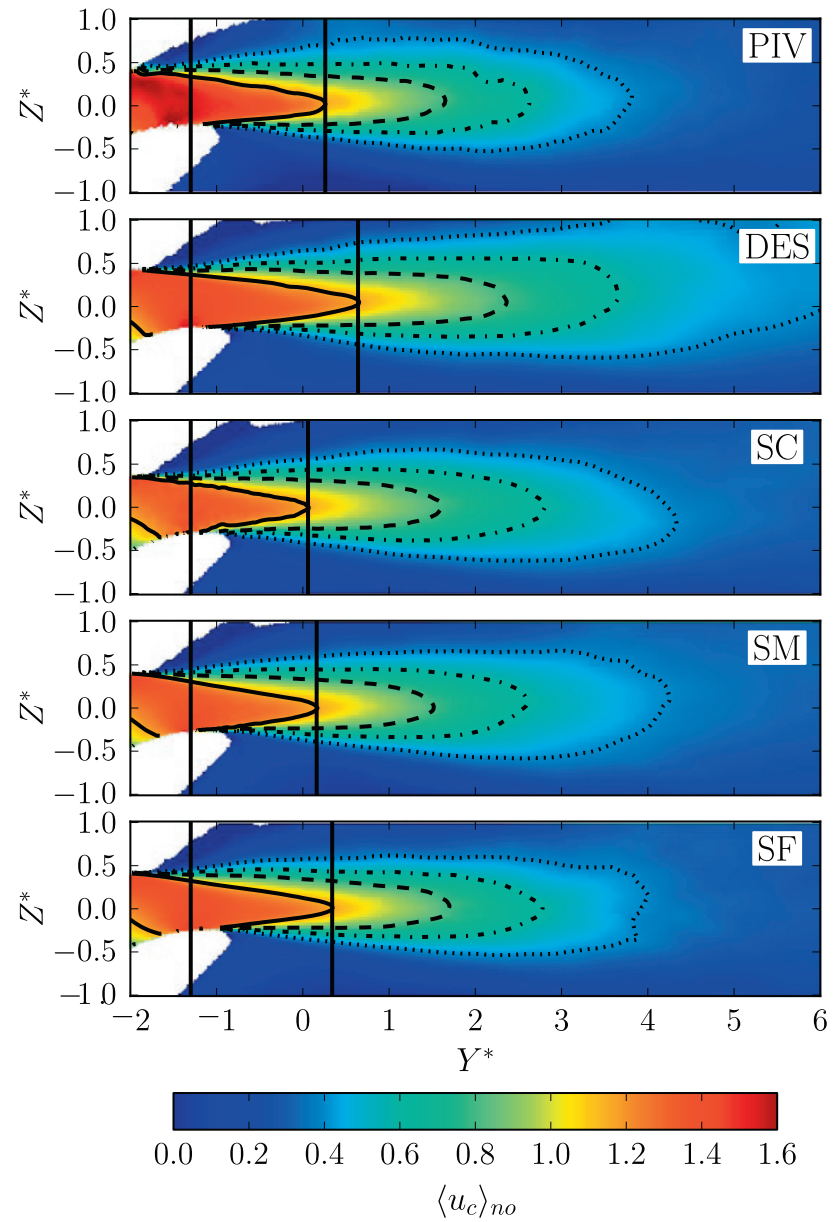

Figure 17

Normalized ensemble-averaged velocity in the direction of flow in a small sub-area (Fig. 3) obtained by PIV, DES-SST (DES), SC, SM and SF. Iso-contours defined by $\left\langle u_{c}\right\rangle_{\mathrm{no}}$ : $\left\langle u_{c}\right\rangle_{\mathrm{no}}=1.2(-) ;\left\langle u_{c}\right\rangle_{\mathrm{no}}=0.8(--) ;\left\langle u_{c}\right\rangle_{\mathrm{no}}=0.6(--)$; $\left\langle u_{c}\right\rangle_{\text {no }}=0.4(\cdots)$. Vertical black lines indicate the end of Zone I (maximum velocity) and Zone II (defined by $\left\langle u_{c}\right\rangle_{\text {no }}=$ $1.2)$, respectively, in terms of the jet centerline.

of the intake jet and the development of moderator gradients within the free shear layer up to $Y^{*}=-1$. The maximum velocities in all simulation results are very similar (between $\left\langle u_{c}\right\rangle_{\mathrm{no}}=1.36$ for $\mathrm{SC}$ and $\left\langle u_{c}\right\rangle_{\mathrm{no}}=1.40$ for DES), but the PIV exhibits increased values $\left(\left\langle u_{c}\right\rangle_{\mathrm{no}}=1.50\right)$. Note that in the valve-gap region is an increased uncertainty in the PIV, which was already illustrated in Figure 12. Downstream of $Y^{*}=0$, transverse gradients in the mean streamwise velocity decrease. In the parabolic velocity profiles, a unique maximum is identified, even though there is still asymmetry. Comparing the simulation and PIV, the SF shows the best agreement, while SC and SM underestimate the maximum velocity. The DES shows the largest deviations in terms of the velocity profile and velocity maximum. The same trend is obtained for $Y^{*}=1$.

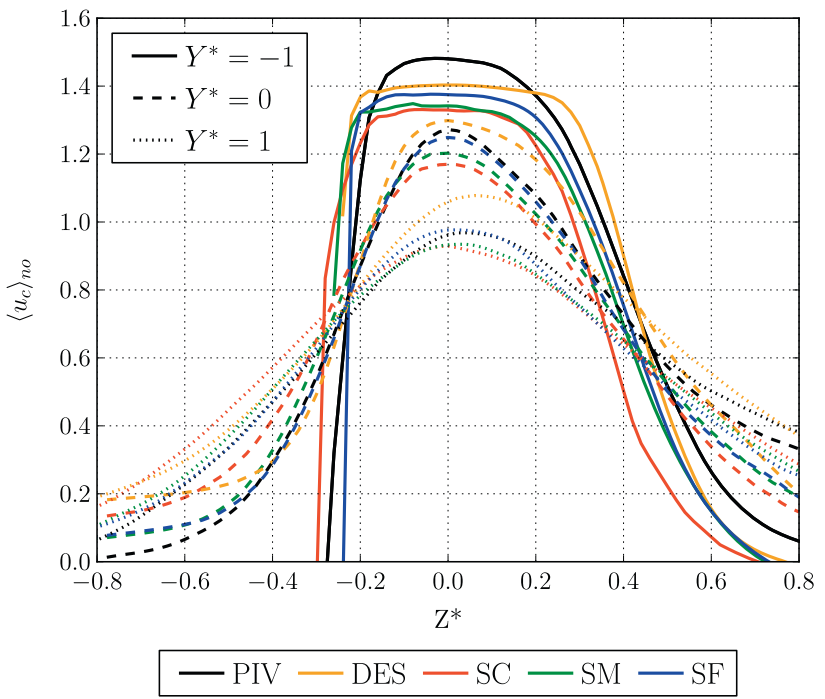

Figure 18

Normalized ensemble-averaged velocity in the direction of flow along the jet cross-section ( $Z^{*}$ axis) at $Y^{*}=-1,0$ and 1 obtained by PIV, DES-SST (DES), SC, SM and SF.

\section{CONCLUSION}

This work investigated a steady-state flow bench configuration, focusing on the flow field in the vicinity of the intake valve. After an preliminary experimental study, the combination of $5 \mathrm{~mm}$ valve lift and $3 \mathrm{~kg} \mathrm{~min}^{-1}$ flow rate was chosen for further investigation by scale-resolving simulations. The DES-SST and the Sigma turbulence model with three different grids (coarse, medium and fine) were applied for this specific operation point. The results were compared to each other and to 2D-2C PIV measurements in terms of the general flow topology, averaged velocity and resolved velocity fluctuations. An ad-hoc methodology was used to extract the centerline of the intake jet and to investigate its position and penetration depth. The results are summarized as follows:

1. Preliminary experimental study:

The intake jet's velocity field varies strongly with the mass flow (valve gap Reynolds number $R e$ ) and the valve lift. The combination of a small $R e$ and a small valve lift, representing the early or late phase of an intake stroke or operation with reduced maximum valve lift, results in an intake jet which is oriented towards the cylinder head. In contrast, the intake jet is oriented towards the center of the cylinder at high $R e$ and small valve lifts.

2. Flow structure at the specified operation point:

The valve center plane (the plane imaged by PIV), intersects the conical intake jet in two places $\left(C_{L}\right.$ and $C_{R}$ ). The jet $C_{L}$ is deflected downwards by the cylinder wall. The jet $C_{R}$ curves downward and part of this flow 
forms a large recirculation zone below the valve, in the center of the combustion chamber.

3. Qualitative and quantitative comparison:

The results of the experiment and all simulations exhibit a significant level of resolved small-scale structures close to the valve gap and throughout the cylinder. The ensemble-averaged flow fields are very similar, but there are differences in the orientation and penetration depth of the intake jet as well as the position of the recirculation zone. A quantitative comparison showed that the orientation and penetration depth of the intake jet $C_{R}$ differs most between PIV and DES. Also, the PIV showed increased velocity fluctuations at $C_{R}$ and the center of cylinder.

4. Detailed analysis of the intake jet:

The mean in-plane location of the jet centerline was estimated in experiment and simulation. This analysis showed that small differences within the valve gap amplify along the direction of flow. The curvature of the intake jet shows a significant grid sensitivity, where the Sigma model on the fine mesh shows best agreement compared to the experiment. For a more detailed comparison, the velocity field was transformed into a component in the direction of the jet centerline and a component normal to it. Based on the velocity component aligned with the jet centerline, three zones could be identified: acceleration, moderate velocity decrease and strong velocity decrease. The intake jet's penetration depth was evaluated by the location on the jet centerline where the mean velocity has decreased to $60 \%, 40 \%, 30 \%$ and $20 \%$ of the characteristic velocity, $v_{\text {char }}$. In this metric, the results obtained by SF agree best with the PIV. In contrast, the DES overpredicts the penetration depth, and differences were identified in the spreading of the intake jet.

In summary, the results obtained by the different turbulence models are very similar and close to the experimental results, while there are differences at specific points (see above). For the DES model, the flow in the vicinity of the valve gap seems to be influenced by the URANS approach. The instantaneous velocity fields of the Sigma model show an increasing amount of resolved turbulent structures with increasing grid points. For this flow, the Sigma model on the medium mesh appears to be a reasonable compromise between accuracy and cost.

\section{ACKNOWLEDGMENTS}

The authors kindly acknowledge the financial support by the Forschungsvereinigung Verbrennungsmotoren (FVV) and the Deutsche Forschungsgemeinschaft (DFG) in the project "BSZ II" (FVV project number 6011333, DFG project ID Schu 1369/11-2) and the Rückkehrerprogramm of the
NRW Ministry for Innovation, Science, and Research. Computer resources on the supercomputer Taurus have been provided by the $Z I H$ at $T U$ Dresden under the grant number p_icengine. Licences were provided by ANSYS Germany.

\section{REFERENCES}

1 Borée J., Miles P.C. (2014) In-cylinder flow, in: Crolla D., Foster D., Kobayashi T., Vaughan N. (eds), Encyclopedia of Automotive Engineering, John Wiley \& Sons, Ltd.

2 Hasse C. (2016) Scale-resolving simulations in engine combustion process design based on a systematic approach for model development, Int. J. Engine Res. 17, 1, 44-62.

3 Borée J., Maurel S., Bazile R. (2002) Disruption of a compressed vortex, Phys. Fluids 14, 7, 2543-2556.

4 Hasse C., Sohm V., Durst B. (2009) Detached eddy simulation of cyclic large scale fluctuations in a simplified engine setup, Int. J. Heat Fluid Flow 30, 1, 32-43.

5 Buhl S., Gleiss F., Köhler M., Hartmann F., Messig D., Bücker C., Hasse C. (2016) A combined numerical and experimental study of the 3D tumble structure and piston boundary layer development during the intake stroke of a gasoline engine, Flow Turbul. Combust. 98, 579-600.

6 Sjunnesson A., Nelsson C., Max E. (1991) LDA measurements of velocities and turbulence in a bluff body stabilized flame, Laser Anemometry Adv. Appl. 3, 83-90.

7 Lyn D., Einav S., Rodi W., Park J. (1995) A laser-Doppler velocimetry study of ensemble-averaged characteristics of the turbulent near wake of a square cylinder, J. Fluid Mech. 304, 285-319.

8 Kravchenko A., Moin P. (2000) Numerical studies of flow over a circular cylinder at $\operatorname{Re}_{\mathrm{D}}=3900$, Phys. Fluids 12, 2, 403-417.

9 Armalyt B., Durst F., Pereira J., Schönung B. (1983) Experimental and theoretical investigation of backward-facing step flow, J. Fluid Mech. 127, 473-496.

10 Jovic S., Driver D. (1994) Backward-facing step measurements at low Reynolds number, $\mathrm{Re}_{\mathrm{h}}=5000$, Technical report, NASA, USA, Available on https://ntrs.nasa.gov/search.jsp? $\mathrm{R}=19940028784$.

11 Le H., Moin P., Kim J. (1997) Direct numerical simulation of turbulent flow over a backward-facing step, J. Fluid Mech. 330, 1, 349-374.

12 Nasr A., Lai J. (1998) A turbulent plane offset jet with small offset ratio, Exp. Fluids 24, 1, 47-57.

13 Assoudi A., Habli S., Saïd N., Bournot H., Le Palec G. (2015) Experimental and numerical study of an offset jet with different velocity and offset ratios, Eng. Appl. Comp. Fluid Mech. 9, 1, 490-512.

14 Klein M., Sadiki A., Janicka J. (2003) Investigation of the influence of the Reynolds number on a plane jet using direct numerical simulation, Int. J. Heat Fluid Flow 24, 6, 785-794.

15 Amamou A., Habli S., Sad N., Bournot P., Le Palec G. (2015) Numerical study of turbulent round jet in a uniform counterflow using a second order Reynolds stress model, J. Hydro-Environ. Res. 9, 4, 482-495.

16 Lübcke H., Schmidt S., Rung T., Thiele F. (2001) Comparison of LES and RANS in bluff-body flows, J. Wind Eng. Ind. Aerodyn. 89, 14-15, 1471-1485. 
17 Catalano P., Wang M., Iaccarino G., Moin P. (2003) Numerical simulation of the flow around a circular cylinder at high Reynolds numbers, Int. J. Heat Fluid Flow 24, 4, 463-469.

18 Hasse C., Sohm V., Wetzel M., Durst B. (2009) Hybrid URANS/LES turbulence simulation of vortex shedding behind a triangular flameholder, Flow Turbul. Combust. 83, $1,1-20$.

19 Cavar D., Meyer K. (2012) LES of turbulent jet in cross-flow: part 1-A numerical validation study, Int. J. Heat Fluid Flow 36, 18-34.

20 Akselvoll K., Moin P. (2014) Large eddy simulation of a backward facing step flow, Eng. Turbul. Model. Exp. 2, 303-313.

21 Hartmann F., Buhl S., Gleiss F., Barth P., Schild M., Kaiser S.A., Hasse C. (2016) Spatially resolved experimental and numerical investigation of the flow through the intake port of an internal combustion engine, Oil Gas Sci. Technol. - Rev. IFP 71, 1, 2.

22 Freudenhammer D., Baum E., Peterson B., Böhm B., Jung B., Grundmann S. (2014) Volumetric intake flow measurements of an IC engine using magnetic resonance velocimetry, Exp. Fluids 55, 5, 1-18.

23 Voisine M., Thomas L., Borée J., Rey P. (2011) Spatio-temporal structure and cycle to cycle variations of an in-cylinder tumbling flow, Exp. Fluids 50, 5, 1393-1407.

24 Baum E., Peterson B., Böhm B., Dreizler A. (2014) On the validation of LES applied to internal combustion engine flows: part 1: comprehensive experimental database, Flow Turbul. Combust. 92, 1-2, 269-297.

25 Zentgraf F., Baum E., Böhm B., Dreizler A., Peterson B. (2016) On the turbulent flow in piston engines: coupling of statistical theory quantities and instantaneous turbulence, Exp. Fluids 28, 4.

26 Mi J., Deo R., Nathan G. (2005) Characterization of turbulent jets from high-aspect-ratio rectangular nozzles, Phys. Fluids 17,6 .

27 Muppidi S., Mahesh K. (2008) Direct numerical simulation of passive scalar transport in transverse jets, J. Fluid Mech. 598, 335-360

28 Jones E., Oliphant T., Peterson P. (2001) SciPy: open source scientific tools for Python, http://www.scipy.org/.

29 Melling A. (1997) Tracer particles and seeding for particle image velocimetry, Meas. Sci. Technol. 8, 12, 1406-1416.

30 Freudenhammer D., Peterson B., Ding C., Böhm B., Grundmann S. (2015) The Influence of cylinder head geometry variations on the volumetric intake flow captured by magnetic resonance velocimetry, SAE Int. J. Engine 8, 4, 1826-1836.

31 Nicoud F., Toda H., Cabrit O., Bose S., Lee J. (2011) Using singular values to build a subgrid-scale model for large eddy simulations, Phys. Fluids 23, 8.

32 Rieth M., Proch F., Stein O., Pettit M., Kempf A. (2014) Comparison of the Sigma and Smagorinsky LES models for grid generated turbulence and a channel flow, Comput. Fluids 99, 172-181.
33 Travin A., Shur M., Strelets M., Spalart P. (2002) Physical and numerical upgrades in the detached-eddy simulation of complex turbulent flows, in: Friedrich R., Rodi W. (eds), Advances in LES of Complex Flows, The Netherlands, Springer, pp. 239-254.

34 Hasse C., Sohm V., Durst B. (2010) Numerical investigation of cyclic variations in gasoline engines using a hybrid URANS/LES modeling approach, Comput. Fluids 39, 1, 25-48.

35 Spalart P., Jou W., Strelets M., Allmaras S. (1997) Comments on the feasibility of LES for wings, and on a hybrid RANS/LES approach, $A d v . D N S / L E S$ 1, 4-8.

36 Wilcox D. (1994) Turbulence modeling for CFD, Vol. 2, DCW Industries Inc, La Canada, California, USA.

37 Menter F. (1994) Two-equation eddy-viscosity turbulence models for engineering applications, AIAA J 32, 8, 1598-1605.

38 Hanjalié K., Popovac M., Hadžiabdié M. (2004) A robust nearwall elliptic-relaxation eddy-viscosity turbulence model for CFD, Int. J. Heat Fluid Flow 25, 6, 1047-1051.

39 Jainski C., Lu L., Dreizler A., Sick V. (2013) High-speed micro particle image velocimetry studies of boundary-layer flows in a direct-injection engine, Int. J. Engine Res. 14, 3, 247-259.

40 Raithby G., Schneider G. (1979) Numerical solution of problems in incompressible fluid flow: treatment of the velocity-pressure coupling, Numer. Heat Transfer 2, 4, 417-440.

41 Van Doormaal J., Raithby G. (1984) Enhancements of the simple method for predicting incompressible fluid flows, Numer. Heat Transfer 7, 2, 147-163.

42 Raw M. (1996) Robustness of coupled algebraic multigrid for the Navier-Stokes equations, 34th Aerospace Sciences Meeting and Exhibit, Aerospace Sciences Meetings, Reno, NV, USA, Jan. 1996, AIAA Paper 96-0297, p. 297.

43 Rhie C., Chow W. (1983) Numerical study of the turbulent flow past an airfoil with trailing edge separation, AIAA $J$ 21, 11, 1525-1532.

44 Buhl S., Hartmann F., Hasse C. (2016) Identification of large-scale structure fluctuations in IC engines using pod-based conditional averaging, Oil Gas Sci. Technol-Rev. IFP 71, 1, 1 .

45 Merziger G. (2010) Formeln + Hilfen Höhere Mathematik, Binomi, Barsinghausen, Germany.

46 Dierckx P. (1982) Algorithms for smoothing data with periodic and parametric splines, Comput. Graph. Image Process. 20, 2, 171-184.

47 Sforza P., Trentacoste N. (1967) Further experimental results for three-dimensional free jets, AIAA J 5, 5, 885-891.

48 Maurel S., Solliec C. (2001) A turbulent plane jet impinging nearby and far from a flat plate, Exp. Fluids 31, 6, 687-696.

Manuscript submitted in November 2016

Manuscript accepted in April 2017

Published online in June 2017 